\title{
Nonlinear dynamics in superlattices driven by high frequency ac-fields
}

\author{
J. Devis and E. Diez \\ GISC, Departamento de Matemáticas, Universidad Carlos III, E-28911 Leganés, Madrid, Spain \\ V. Bellani \\ INFM-Dipartimento di Fisica "A. Volta”, Universitá di Pavia, I-27100 Pavia, Italy \\ (May 15, 2017)
}

\begin{abstract}
We investigate the dynamical processes taking place in nanodevices driven by high-frequency electromagnetic fields. We want to elucidate the role of different mechanisms that could lead to loss of quantum coherence. Our results show how the dephasing effects of disorder that destroy after some periods coherent oscillations, such as Rabi oscillations, can be overestimated if we do not consider the electron-electron interactions that can reduce dramatically the decoherence effects of the structural imperfections. Experimental conditions for the observation of the predicted effects are discussed.
\end{abstract}

Typeset using REVTEX 
Recent advances in laser technology make possible to drive semiconductor nanostructures with intense coherent ac-dc fields. This opens new research fields in time-dependent transport in mesoscopic systems [1] and puts forward the basis for a new generation of ultrahigh speed devices. Following these results several works have been devoted to the analysis of the effects of time-dependent fields on the transport properties of resonant heterostructures 2 4, and to exploit the application in ultrafast optical technology: high-speed optical switches, coherent control of excitons, etc. [5, [6].

Within this context, we are interested in the decoherence processes producing the observed fast dephasing of coherence phenomena in semiconductor superlattices (SL's) and more specifically in the interplay between the growth imperfections (disorder) and manybody effects as the electron-electron (e-e) interaction. The interplay between the effects of disorder and many-body effects on electronic properties is a long-standing problem in solid-state physics. Probably one of the most promising way to gain insight into this intricate problem is to combine the actual state-of-the-art of the Molecular Beam Epitaxy (MBE), which allow us to grew samples with monolayer perfection and consequently with well-characterized disorder, with coherent oscillations that are extremely sensitive to imperfections and nonlinear effects.

The oscillations of a two level system between the ground and excited states in the presence of a strong resonant driving field, often called transient nutation or Rabi oscillation (RO), are discussed in textbooks as a topic of time-dependent perturbation theory. Consider a two state system with ground state energy $E_{0}$ and excited state $E_{1}$ in the presence of a harmonic perturbation. If the frequency of the perturbation matches roughly the spacing between the two levels, the system undergoes oscillations with a frequency $\Omega_{R}$ which is much smaller than the excitation frequency $\omega_{\mathrm{ac}}$. This Rabi frequency depends on the mismatch $\delta \omega \equiv\left(E_{1}-E_{0}\right) / \hbar-\omega_{\mathrm{ac}}$ between the level spacing and the excitation frequency, and on the matrix element $F_{10}$ of the perturbation $\Omega_{R}=\left(\delta \omega^{2}+\left|F_{10}\right|^{2} / \hbar^{2}\right)^{1 / 2}$. If we start with the system initially in the ground state, transitions between the ground and the excited state will occur with a period $T_{R}=2 \pi / \Omega_{R}$ as time evolves. 
Semiconductor SL's present Bloch minibands with several states each one; thus it is not clear whether they can be correctly described as a a pure two state system. We should also take into account the presence of imperfections introduced during growth processes and scattering mechanism as e-e interactions. Interface roughness appearing during growth in actual SL's depends critically on the growth conditions. For instance, protrusions of one semiconductor into the other cause in-plane disorder and break translational invariance parallel to the layers. To describe local excess or defect of monolayers, we allow the quantum well widths to fluctuate uniformly around the nominal values; this can be seen as substituting the nominal sharp width by an average along the parallel plane of the interface imperfections. Our approximation is valid whenever the mean-free-path of electrons is much smaller than the in-plane average size of protrusions as electrons only see micro-quantum-wells with small area and uniform thickness. Therefore, in the following we will take the width of the $n$th quantum well to be $a\left(1+W \epsilon_{n}\right)$, and the width of the $n$th barrier as $b\left(1+W \epsilon_{n}\right)$ where $W$ is a positive parameter measuring the maximum fluctuation, $\epsilon_{n}$ 's are distributed according to a uniform probability distribution, $\mathcal{P}\left(\epsilon_{n}\right)=1$ if $\left|\epsilon_{n}\right|<1 / 2$ and zero otherwise, $a$ is the nominal quantum well width and $b$ is the nominal quantum barrier width. Even with its rather satisfactory degree of success, many-body calculations have difficulties that, in some cases, may complicate the interpretation of the underlying physical processes. Presilla et al. [7] suggested a new treatment of these interactions that, loosely speaking, could be regarded as similar to Hartree-Fock and other self-consistent techniques, which substitute many-body interactions by a nonlinear effective potential. More recently [8] we have proposed a new model where the nonlinear interaction is driven by a local field instead of the mean-field approach used by Presilla and co-workers. [7].

Here we present a new model that solves self-consistently the Poisson and the timedependent Schödinger equations, and we compare the results with a local treatment based in the non-linear Schrödinger equation.

The band structure and the potential at flat band is computed by using a finite-element method. The eigenstate $j$ of the band $i$ with eigenenergy $E_{i}^{(j)}$ is denoted as $\psi_{i}^{(j)}(x)$. A good 
choice for the initial wave packet is provided by using a linear combination of the eigenstates belonging to the first miniband. For the sake of clarity we have selected as the initial wave packet $\Psi(x, 0)=\psi_{i}^{(j)}(x)$, although we have checked that this assumption can be dropped without changing our conclusions. The subsequent time evolution of the wave packet $\Psi(x, t)$ is calculated numerically by means of an implicit integration schema designed for consider time-dependent fields [9].

The envelope-functions for the electron wavepacket satisfies the following quantum evolution equation

$$
i \hbar \frac{\partial \Psi(x, t)}{\partial t}=\left[-\frac{\hbar^{2}}{2 m^{*}} \frac{d^{2}}{d x^{2}}+V_{N L}(x, t)\right] \Psi(x, t),
$$

where $x$ is the coordinate in the growth direction of the SL. We consider two approaches to the nonlinear potential $V_{N L}(x, t)$ in Eq.(1). On the one hand we take into account the model described, in other context, in [8] for our problem, where $V_{N L}(x, t)$ is

$$
V_{N L}(x, t)=V(x)-e F_{A C} x \sin \left(\omega_{A C} t\right)+\alpha_{l o c}|\Psi(x, t)|^{2},
$$

and $V(x)$ is the potential at flat-band, $F_{A C}$ and $\omega_{A C}$ are the strength and the frequency of the ac field respectively, and all the nonlinear physics is contained in the coefficient $\alpha_{l o c}$, which we discuss below. There are several factors that configure the medium nonlinear response to the tunneling electron. We want to consider only the repulsive electron-electron Coulomb interactions, which should enter the effective potential with a positive nonlinearity, i.e., the energy is increased by local charge accumulations, leading to a positive sign for $\alpha_{l o c}$.

On the other hand, we have considered a different approach by solving self-consistently the Schrödinger and Poisson equations obtaining a Hartree-like potential. In this context, the non-linear potential is,

$$
V_{N L}(x, t)=V(x)-e F_{A C} x \sin \left(\omega_{A C} t\right)+\alpha_{s e l f} V_{H}(x, t),
$$

where now $V_{H}$ it is obtained by solving the Poisson equation for the density of charge $|\Psi(x, t)|^{2}$, and $\alpha_{\text {self }}$ is the coupling parameter. 
We present here results for a SL with 10 periods of $100 \AA$ GaAs and $50 \AA \mathrm{Ga}_{0.7} \mathrm{Al}_{0.3} \mathrm{As}$ with conduction-band offset $300 \mathrm{meV}$ and $m^{*}=0.067 m, m$ being the free electron mass. To illustrate the effects of the nonlinear coupling we show in Fig. 1 the conduction-band profile for a perfect SL $(W=0)$ at $t=0.4$ (lower) and $1.2 \mathrm{ps}$ (upper) when the ac field is tuned to the resonant frequency $\omega_{\mathrm{ac}}=\omega_{\text {res }} \sim 24 \mathrm{THz}$, for (a) the linear case and modeling the e-e interaction with (b) the self-consistent method $\left(\alpha_{\text {self }}=10^{-3}\right)$ and with (c) the local model $\left(\alpha_{l o c}=10\right)$. To show the effects of the interface roughness we plot in Fig. 2 the probability of finding an electron, initially situated in $\psi_{0}^{(5)}(x)$, in the state $\psi_{1}^{(5)}(x)$ as a function of time when the ac field is tuned to the resonant frequency $\omega_{\text {ac }}=\omega_{\text {res }} \sim 24 \mathrm{THz}$ with (a) $W=0$ (perfect SL) and (b) $W=0.03$ (imperfections around one monolayer). These results suggest the existence of a characteristic scattering time $\tau_{\text {dis }}$ related to the amount of disorder in the sample, after which RO's are destroyed by disorder. In Fig. 3 we plot the probability of finding an electron, initially situated in $\psi_{0}^{(5)}(x)$, in the state $\psi_{1}^{(5)}(x)$ as a function of time when the ac field is tuned to the resonant frequency, for different values of the nonlinearity coupling (a) $\alpha_{\text {self }}=5 \times 10^{-5}$, (b) $10^{-4}$, (c) $5 \times 10^{-4}$ and (d) $10^{-3}$. The results for the local model are very similar. When we compare this picture with Fig. 2 we see the process of vanishing of the RO's are completely different. In the second case, the effects are the same for any time, then we could not speak about a dephasing time, apparently we only modified the electronic structure and then we are decreasing the resonant coupling between the external ac-field and the Bloch bands. Nevertheless the main goal of this work is to show how the nonlinearity effects can reduce the dephasing effects introduced by the growth imperfections. In Fig. 1 we plot the occupation probability of the state $\psi_{1}^{(5)}(x)$ as a function of time considering imperfections about one monolayer ( $W=0.03$ ) for (a) the linear case and considering together with the imperfections the e-e interaction with the selfconsistent model (b) $\alpha_{\text {self }}=10^{-4}$ and with the local one (c) $\alpha_{l o c}=5$. We can see clearly how nonlinearity prevents the dephasing effects introduced by the imperfections allowing the observation of Rabi oscillations during larger coherence times. These theoretical results 
are completely consistent with recent experiments in transport properties of intentional disordered superlattices with doped and undoped superlattices [10,11, where is showed than the Coulomb interactions could be the responsible of the suppression of disorder effects leading to quasimetallic behavior at low temperatures when the doping of the samples is increased.

In summary, we have shown how the dephasing effects of disorder are dramatically reduced when we consider the e-e interaction. We have studied two different models to introduce the non-linear interaction and the results are very similar. Our results shows that it is possible to enlarge the dephasing times and, consequently the number of periods of coherence oscillations of electrons in SL's. In semiconductor heterostructures this can be done by increasing the doping or with very intense laser excitation fields. It goes without saying that to develop new devices for $\mathrm{THz}$ science it is crucial to understand how to control and enlarged the coherence times. We think that the nonlinear effects could be the key to solve this problem. Further work along these lines is currently in progress.

The authors would like to thank Francisco Domínguez-Adame for helpful discussions and critical reading of the manuscript. Also we thank to I. Bossi, Rafael Gómez-Alcalá, Claudio Andreani and Gennady Berman, for very valuable conversations. J.D. and E.D. thanks the Dipartimento di Fisica "A. Volta" of the Universitá di Pavia for hospitality during a stay when part of this work was done. Work in Madrid was supported by CAM under Project No. 07N/0034/1998, and in Pavia by the INFM Network "Fisica e Tecnologia dei Semiconduttori III-V". J.D. and E.D. also acknowledges partial support from Fundación Universidad Carlos III (Spain) and INFM (Italy). 


\section{REFERENCES}

[1] S. T. Cundiff, A. Knorr, J. Feldmann, S. W. Koch, E. O. Göbel and H. Nickel, Phys. Rev. Lett. 73, 1178 (1994).

[2] E. Diez, R. Gómez-Alcalá, F. Domínguez-Adame, A. Sánchez and Gennady P. Berman, Phys. Lett. A 240, 109 (1998).

[3] E. Diez, R. Gómez-Alcalá, F. Domínguez-Adame, A. Sánchez and Gennady P. Berman, Phys. Rev. B 58, 1146 (1998).

[4] E. Diez, F. Domínguez-Adame, A. Sánchez, Microelectronic Engineering, 43, 103 (1998).

[5] D. S. Citrin, Phys. Rev. Lett. 77, 4596 (1996).

[6] D. S. Citrin, Appl. Phys. Lett. 70, 1189 (1997).

[7] C. Presilla, G. Jona-Lasinio, and F. Capasso, Phys. Rev. B 43, 5200 (1991).

[8] E. Diez, A. Sánchez and F. Domínguez-Adame, Phys. Lett. A 215, 103 (1996).

[9] Details about the numerical procedure and algorithms will be given elsewhere.

[10] G. Ritcher, W. Stolz, P. Thomas, S. W. Kock, K. Maschke, and I. P. Zvyagin, Superlattices and Microstructures 22, 475 (1997).

[11] V. Bellani, E. Diez, R. Hey, L. Toni, L. Tarricone, G. B. Parravicini, F. DomínguezAdame, and R. Gómez-Alcalá. (submitted)

Available at: http://valbuena.fis.ucm.es/caste/artframe.html 


\section{FIGURES}

FIG. 1. Conduction-band profile.

FIG. 2. The probability of finding the electron in the state $\psi_{1}^{(5)}(x)$ as a function of time for different amounts of disorder.

FIG. 3. The probability of finding the electron in the state $\psi_{1}^{(5)}(x)$ as a function of time for different values of the nonlinear coupling.

FIG. 4. The probability of finding the electron in the state $\psi_{1}^{(5)}(x)$ as a function of time considering the interplay between imperfections and nonlinearities. 


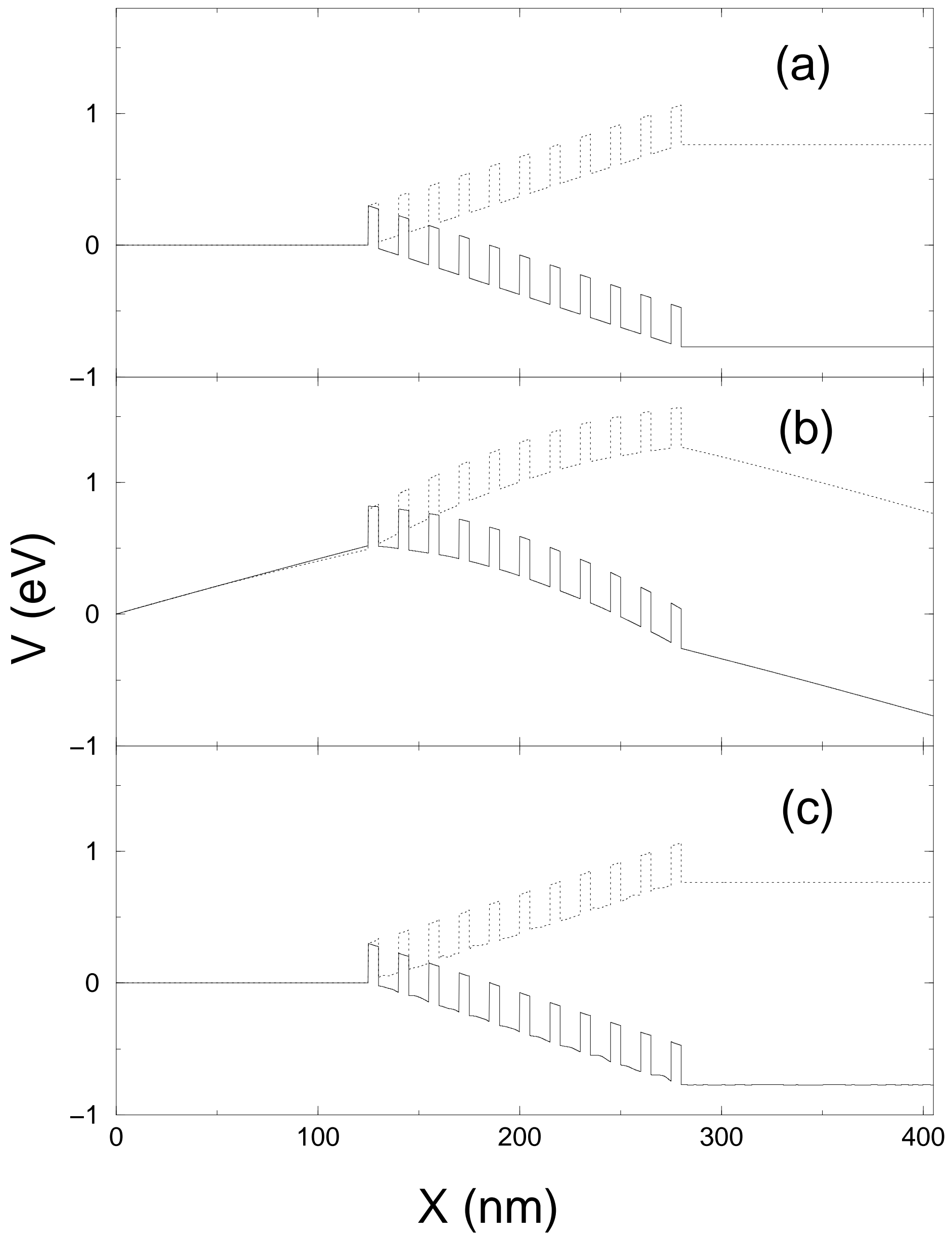




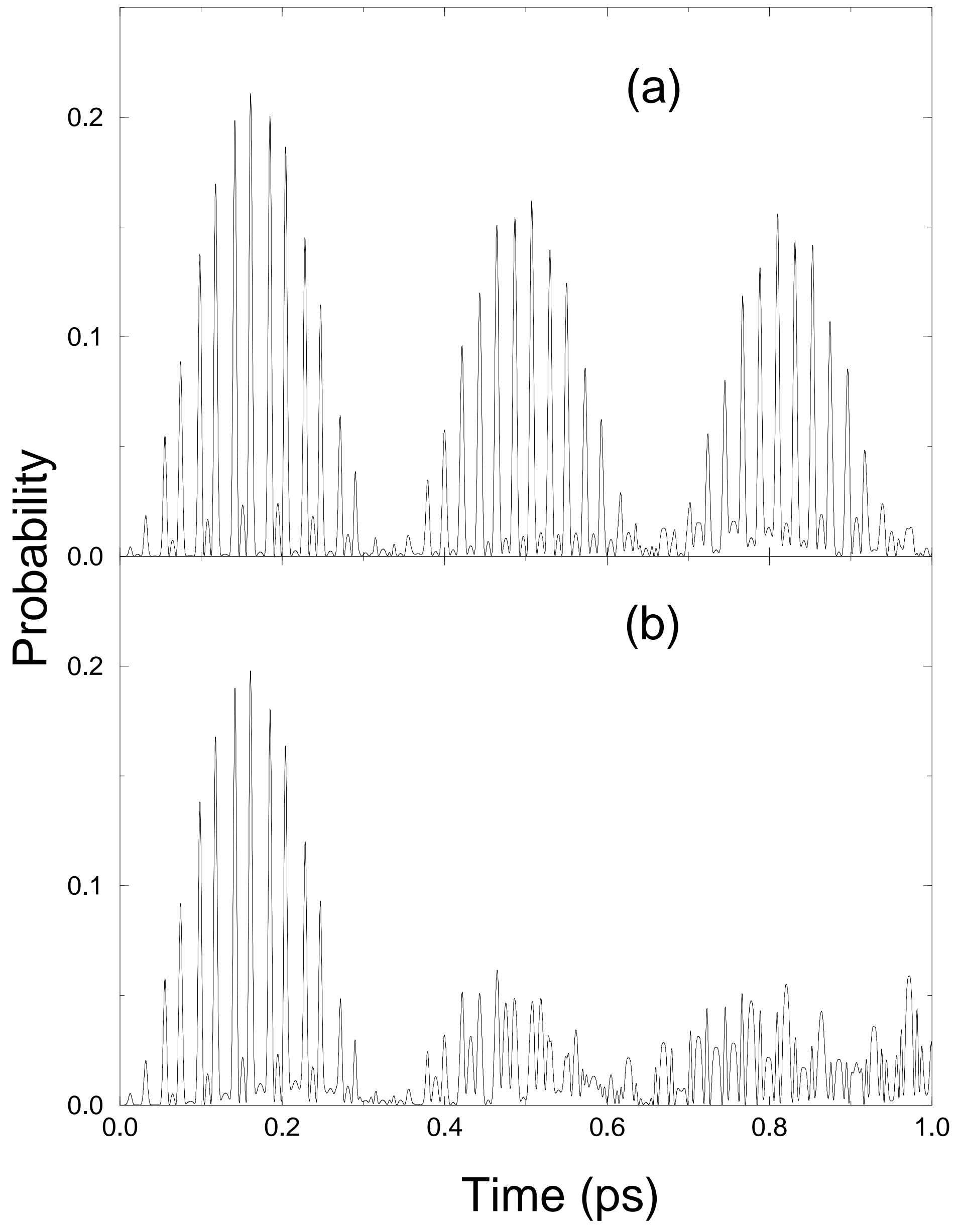




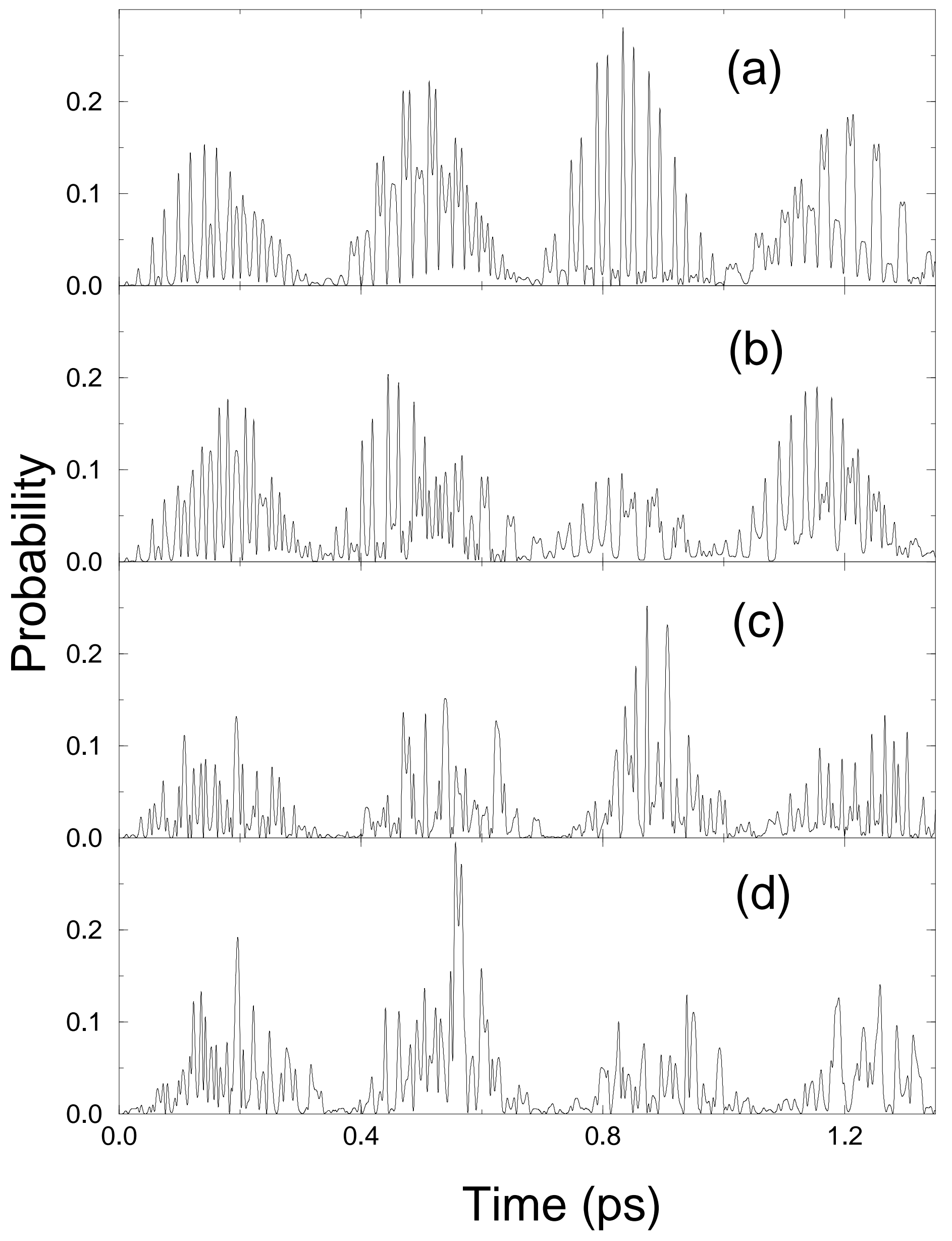




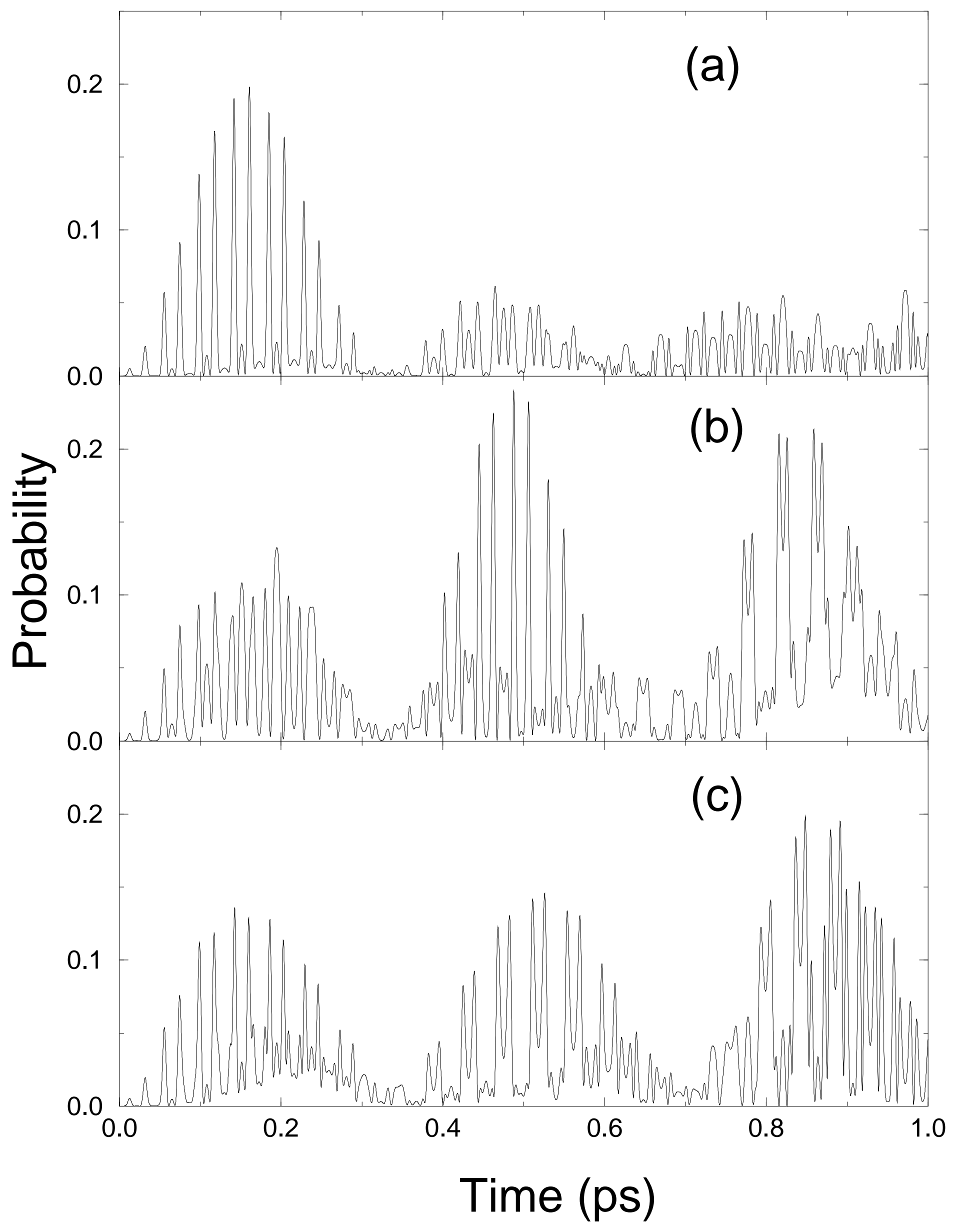

